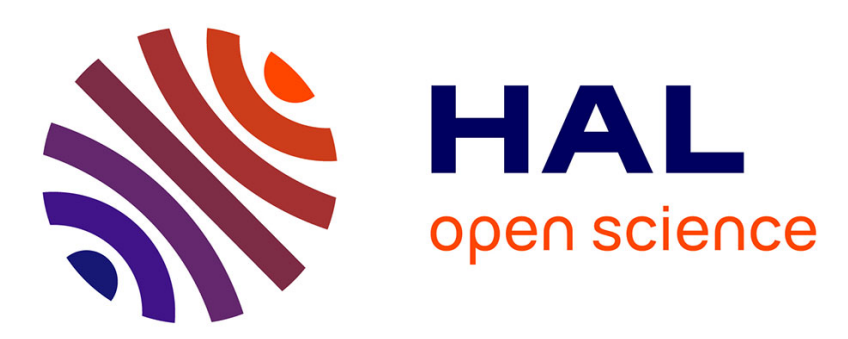

\title{
A behavioral framework for information representation in the brain
}

\author{
Frédéric Alexandre
}

\section{To cite this version:}

Frédéric Alexandre. A behavioral framework for information representation in the brain. Ahmed A. Moustafa. Computational Models of Brain and Behavior, Wiley, pp.403-412, 2017, 978-1-119-15906-3. hal-01651751

\section{HAL Id: hal-01651751 \\ https://hal.inria.fr/hal-01651751}

Submitted on 29 Nov 2017

HAL is a multi-disciplinary open access archive for the deposit and dissemination of scientific research documents, whether they are published or not. The documents may come from teaching and research institutions in France or abroad, or from public or private research centers.
L'archive ouverte pluridisciplinaire HAL, est destinée au dépôt et à la diffusion de documents scientifiques de niveau recherche, publiés ou non, émanant des établissements d'enseignement et de recherche français ou étrangers, des laboratoires publics ou privés. 


\title{
A behavioral framework for information representation in the brain
}

\author{
Frédéric Alexandre ${ }^{1,2,3}$ \\ ${ }^{1}$ Inria, Bordeaux Sud-Ouest Research Centre, France \\ ${ }^{2}$ Labri, UMR 5800, CNRS, University of Bordeaux, Bordeaux INP \\ ${ }^{3}$ IMN, UMR 5293, CNRS, Institute of Neurodegenerative Diseases
}

\begin{abstract}
:
Along evolution, increasingly complex cognitive functions have been attributed to an increasingly complex brain architecture. Nevertheless, the brain remains anchored on an organization dedicated to survival. We believe that keeping this principle in mind is an excellent way to better decipher cerebral mechanisms and corresponding cognitive functions. Accordingly, we describe here the main characteristics and constraints of an intelligent agent learning to survive in an intelligent environment, in terms of information flows and learning principles. On this basis, we propose a framework of description for the architecture of the brain of mammals, organized around four fundamental questions to be answered. These questions define the identity of the goal (what?) and the motivation to choose it (why?), its location (where?) and the way to get it (how?). Then we explain how the main requirements of respondent and operant conditioning can be addressed within this architecture and how it is also compatible with the elaboration of more complex cognitive mechanisms. This can be seen as the validation of this framework to explore how cognitive functions might emerge from cerebral circuits and how they have been made more complex along evolution. It also proposes a systemic view of the brain, useful to develop the cognitive architecture of an intelligent agent exploring autonomously its environment and to propose to machine learning innovative algorithms.
\end{abstract}

Keywords : Learning, cerebral systems, cognitive architecture

\section{Introduction}

Most models in computational neuroscience addressing high level cognitive functions like decision making, perceptual identification or selective attention set the focus on the role of the cortex and minimize the role of subcortical structures (Parvizi, 2009). In addition, emotional and motivational dimensions of these functions are generally underrepresented, not to mention bodily dimensions. Yet, a variety of sources indicate that these dimensions are linked one to another and play an important role in high level cognitive functions.

In short, cerebral circuits responsible for emotional tagging of stimuli associate them to a valence (depending if they are appetitive or aversive, particularly to detect and identify mates, preys and predators) and an value (depending on their intensity), whereas motivational circuits are concerned with the evaluation of the needs or the costs associated to a behavior (Cardinal, Parkinson, Hall, \& Everitt, 2002). It has been specifically shown that bodily data are necessary to estimate such information (Damasio \& Carvalho, 2013), and are related to pain, pleasure, fatigue and other visceral and muscular data (Craig, 2009).

At the origin, such pieces of information are necessary for survival to organize the behavior around such important external stimuli and internal feelings. Less primitive species have higher level goals and have developed higher level cognitive functions but we claim here that these functions are still dependent on this initial substratum and their description is not complete if specified without any reference to these domains. Such considerations have been partly addressed in several modeling domains like Embodied Artificial Intelligence (Pfeifer, Bongard, \& Grand, 2007), Enactivism (Varela, Thompson, \& Rosch, 1991; O'Regan \& Noë, 2001) and more generally Cognitive Science (Gros, 2010).

The goal of this paper is to set the basis for this kind of description in the domain of Computational Neuroscience. This domain is frequently used to explore cognitive functions and it is also prone to focusing on higher level mechanisms without any reference to the more primitive bases. To relate both levels, we propose to draw a systemic model of the cerebral circuitry of 
an intelligent agent learning to exploit its environment, in order to satisfy some internal needs. We will specify more precisely in the forthcoming sections (i) information flows and learning principles at work in such tasks and we will propose (ii) a general framework for information representation in the brain. On this basis, we will discuss (iii) the implications for some cognitive mechanisms. Throughout these descriptions, it will be particularly considered that more evolved cerebral mechanisms can be seen as extensions of more elementary survival mechanisms, stressing the need to include the latter ones in the study of advanced cognitive functions.

The main arguments of this picture are inspired from data in neuroscience related to experimentations with primates, which are the main goals of this modeling framework but information from rodents and of course from non mammals will be also considered.

\section{Information processing by the intelligent agent}

The central goal of the nervous system is to help animals maintaining their organic structure Laborit 1976). This is obvious when an animal learns to get some food or to avoid a predator. In this case, we propose to define here the kind of information flows that have to be associated and some learning principles generally observed in such circumstances.

\subsection{Information flows}

We define three worlds (cf. figure 1) that the brain can perceive and on which it can act. The external environment includes objects and agents in the surrounding world, that can be perceived by external sensors (eyes, ears, skin, tongue, nose), defining exteroception. The external body is carrying external sensors and can act in the external environment, depending on its position perceived by proprioception. The internal body corresponds to the visceral, chemical and hormonal machinery of the body that can be perceived by interoception. Homeostasis but also internal and external actions can modify these internal states.

Then brain can perceive these three worlds and build from them a neuronal representation. This representation can be seen as a fourth world that the brain might perceive (depending on cerebral connectivity) and on which it can act, modifying neuronal activations. Some of them will have an impact on the other three worlds and will be considered as actions at large, modifying internal states, position of (parts of) the body or the surrounding world itself.

\subsection{Learning principles}

All the actors of the picture evoked above are subject to the laws of physics or to the laws of nature including intentionality. There are consequently regularities and invariants that can be extracted, even if the bad quality of perception and action (and possibly other reasons) makes often the world appear as partly stochastic and unknown. Extracting and exploiting these regularities (and adapting to uncertainty) is the main purpose of learning.

Evolution is sometimes considered as a kind of learning with a very slow constant of time, leading to the selection of some information flows and of some causal relations between the resulting representations, which can be called homeostasis or reflexes. Nevertheless, learning is preferentially described as the activity dependent plasticity of the neuronal circuitry, yielding the elaboration of different kinds of memory, to represent and store regularities extracted under a variety of learning processes. Emotional learning is related to respondent (or pavlovian) conditioning (Balleine \& Killcross, 2006) and is organized around the detection of unconditional stimuli US, some biologically significant stimuli (like the perception of food or predators) triggering automatic and reflexe responses (like salivation or freezing). The goal of pavlovian conditioning is to learn to associate a US to a conditional stimulus CS that announces it. Depending on the distance (in space and time) to these stimuli, this can generate a consummatory phase to get prepared to the US or a preparatory phase more related to orientation, corresponding in both cases to a stereotyped behavior.

In summary, pavlovian conditioning performs mainly an emotional tagging of the environment, in order to detect noxious or appetitive stimuli. On this basis, some elementary automatic bodily, hormonal or autonomic responses can be triggered (LeDoux, 2007) to prepare the body to an inevitable event or, at best, to improve its orientation. In contrast, operant (or instrumental) conditioning is going to consider such stimuli as potential external goals to avoid or to reach and to confront them to internal goals corresponding to needs (levels of thirst or hunger) and integrity of the body (risk of harm or fatigue). Motivational learning will correspond to learn in which context to explicitly select actions (including internal actions and decisions) to obtain such reinforcement (goals reaching or avoiding) and will be described as a goal-directed behavior. 


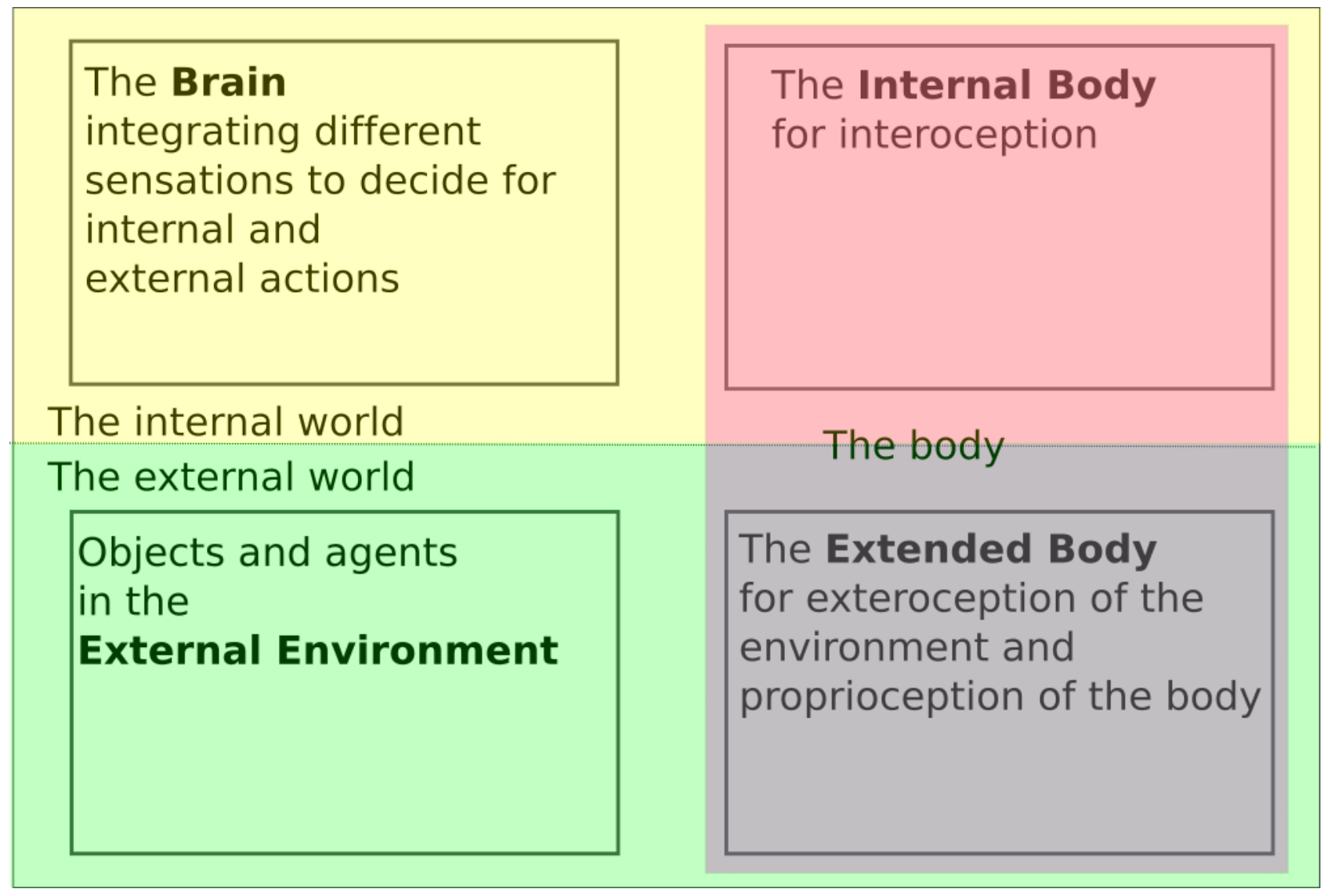

FIGURE 1 - Systemic view of the brain and its relations to the external environment and the body, including its internal and external facet. Perception of these worlds corresponds respectively to exteroception, interoception and proprioception. This results in a neuronal representation which might decide for a modification (considered as an action) in any of these worlds. These information flows are the basis for perceiving needs and goals and for defining the most appropriate responses for the survival of the agent. 
After extensive learning, the context will be sufficient to trigger actions with no reference to the targeted reinforcement and will be called habitual behavior (Gruber \& McDonald, 2012).

In addition to these two learning paradigms, central for survival capabilities and present in all vertebrates (Laberge, Muhlenbrock-Lenter, Grunwald, \& Roth, 2006), other kinds of learning have evolved to increase animal skills, complexifying their semantic, episodic or working memories and accordingly the kind of information they can manipulate, as described below.

\section{A reasoned framework of in- formation representation in the brain}

We have described some characteristics of an animal seen as an agent engaged in emotional and motivational learning for a survival purpose. We can reasonably propose that regularities extracted by these and other kinds of learning will be extracted and represented in the brain from information exchanged with the three worlds enumerated above and with other brain regions. Specifically, these pieces of knowledge will be used to act on and modify these worlds and neuronal activity in the brain. Beyond survival, we will argue below that some of these behaviors are also classically described as mobilizing high level cognitive functions, depending on the mechanisms engaged.

In order to emphasize that this description of the cognitive architecture is not a loose analogy but remains tightly constrained by the cerebral circuitry, we propose here a reasoned framework of information representation in the brain (cf figure 2) and explain how it fits with known anatomical and physiological characteristics of the brain and how it is particularly convenient to bear information flows and learning principles evoked above.

On the one hand, we describe the brain as loops between five major kinds of neuronal structures : (i) Ancient extracortical structures for elementary sensorimotor associations; (ii) The sensory cortex for the representation of sensory information; (iii) The hippocampus for the binding of multimodal information; (iv) The frontal cortex for action representation; (v) The basal ganglia for action selection. On the other hand, we propose to organize the behavior around four fundamental questions that the brain has to answer : What is the goal of the action? Why is it appropriate considering the current motivation? Where is this goal localized? How it can be reached? For each of that questions, we propose an initial level of representation, as generally described in the sensory cortex and in ancient extracortical structures.

What - The representation of the goal : The amygdala is an ancient cerebral structure playing a central role in pavlovian conditioning (LeDoux, 2007). It is composed of several nuclei (Swanson \& Petrovich, 1998), including the basolateral nucleus involved in learning the CS-US association and the central nucleus for the expression of emotional responses and related physiological responses, through structures like the periaqueductal gray (PAG) and the lateral hypothalamus and also through nuclei releasing neuromodulators (LeDoux, 2007). The basolateral region has been extensively studied (Belova, Paton, Morrison, \& Salzman, 2007; Pessoa \& Adolphs, 2010) and appears to encode many characteristics related to the CS and the US. It can be consequently described as involved in the representation of goals or elements of goals of the behavior. This region is also in relation with many cerebral structures informed by emotional learning and able to provide amygdala with more precise and elaborate information than rough sensory information received by the amygdala in primitive animals (Laberge et al., 2006). There are particularly massive relations with the Medial Temporal Lobe, including the medial prefrontal cortex, the hippocampus and the temporal region of the sensory cortex (cf (Carrere \& Alexandre, 2015 for details). Specifically, neurons in the temporal cortex has been described as selectively responding to physical characteristics (Rousselet, Thorpe, \& FabreThorpe, 2004), necessary to describe and identify objects that might correspond to the goal of the behavior when associated to an attentional process.

Why - Motivation of the goal : The lateral hypothalamus is an ancient sensorimotor structure related to the processing of needs, since it is responsive to appetitive US and triggers actions related to digestive functions (Craig, 2003). The same can be said about the PAG, rather concerned with aversive stimuli and defensive behavior (Bandler \& Shipley, 1994). It has also been reported that more complex motivational behavior can be prepared in these structures in collaboration with the posterior insula, a cerebral sensory region known for example to represent levels of water deprivation or precise characteristics of a pain (Craig, 2009), which are typical examples of motivations to act.

Where - Position of the goal : The superior colliculus is a central structure for eye movements and gaze orientation (Lee, Rohrer, \& Sparks, 1988), including sensory layers representing the surrounding 


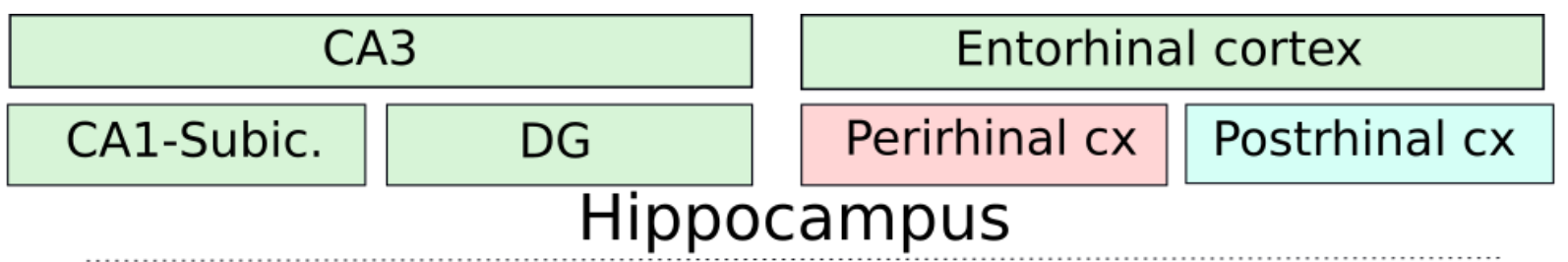

\section{Hippocampus}

Frontal cortex

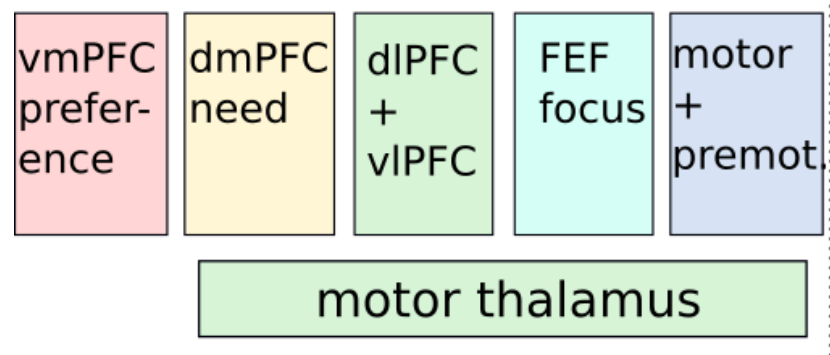

Sensory cortex

\begin{tabular}{|c|c|c|c|c|}
\hline \begin{tabular}{|l|} 
Exteroc \\
eption \\
Temporal \\
What?
\end{tabular} & $\begin{array}{l}\text { Interoc } \\
\text { eption } \\
\text { Insula } \\
\text { Why? }\end{array}$ & $\begin{array}{l}\text { Multi- } \\
\text { modal } \\
\text { assoc. }\end{array}$ & $\begin{array}{l}\text { Location } \\
\text { Parietal } \\
\text { Where? }\end{array}$ & $\begin{array}{l}\text { Proprio } \\
\text { ception } \\
\text { Parietal } \\
\text { How? }\end{array}$ \\
\hline
\end{tabular}

\section{sensory thalamus}

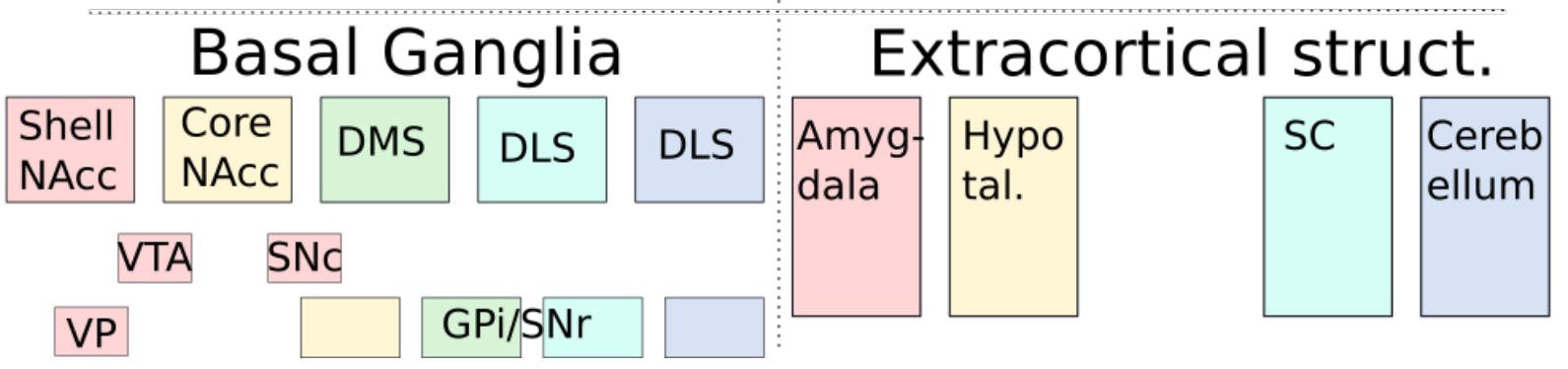

Figure 2 - This scheme proposes to highlight some elements of information representation, extracted from the literature describing the brain of mammals and mostly of primates. Considering the five main cerebral regions (the basal ganglia, the sensory and the frontal cortex, the hippocampus and several ancient extracortical sensorimotor regions taken as a whole), colors in the scheme indicate some important relations between these structures forming loops, even if the text reports also other important connections between loops. In the picture, a simple keyword refers roughly, in the cortical regions, to the kind of fonctions this region contributes and the text explains in some more details that these functions address four fundamental questions (What, Why, Where and How). In the basal ganglia, the striatum is composed of its dorsolateral part DLS, dorsomedial part DMS and limbic part, also called Nucleus Accumbens NAcc with a shell and a core part; with output structures like the internal Globus Pallidus and the substantia nigra pars reticulata GPI-SNr and the ventral pallidum VP; with dopaminergic regions ventral tegmentum area VTA and substantia nigra pars compacta SNc). Several regions of the sensory cortex (in the parietal, temporal and insular parts) are receiving inputs from the sensory thalamus and regions of the frontal cortex include the ventromedial prefrontal cortex vmPFC, the dorsomedial prefrontal cortex dmPFC, the lateral prefrontal cortex with ventral and dorsal parts vlPFC and dlPFC, the frontal eye field FEF and the motor and premotor regions. The hippocampus is represented with its main internal structures dentate gyrus DG, CA3 and CA1 and its input cortical structures. In the extracortical regions, structures are the Amygdala, the Hypothalamus, the Superior Colliculus (SC) and the Cerebellum. 
environment in topographic layers (Taouali, Goffart, Alexandre, \& Rougier, 2015) and motor layers orienting the body toward stimuli selected by competition inside these maps (Dean, Redgrave, \& Westby, 1989). More complex behaviors of spatial orientation can be obtained by collaboration with the Frontal Eye Field (FEF) regions of the frontal cortex (Sommer \& Wurtz 2004 ) and the posterior dorsal cortex. The superior part of this latter region has been described for a long time (Ungerleider \& Mishkin, 1982) as addressing the Where question in the sensory cortex by representing spatial information about the environment.

How - Motor control of the goal : In contrast, the inferior part of the posterior dorsal cortex has been described as answering the question How (Milner \& Goodale, 1995), particularly because it encodes characteristics useful for object manipulation. In this domain, the cerebellum (Manto et al., 2012) is a key cerebral structure for sensorimotor control and participates with the dorsal cortex to complex voluntary movements (Middleton \& Strick, 2000).

\section{Extension to some cognitive mechanisms}

At this stage, each of the elementary sensorimotor associations evoked above can yield a pertinent behavior and is probably sufficient for an animal with limited perception and behavioral repertoire : in an enactive way (O’Regan \& Noë, 2001), each perception can elicit a response adapted to identify the nature of a goal, orient toward this goal and manipulate and consume it to satisfy a need. Nevertheless, in the real life things are not so simple and these elementary sensorimotor associations might be not complex enough, incompatible or might require a more elaborated decision process to go beyond simple survival in a richer environment including uncertainty and a variety of rules and laws. With no claim of exhaustivity, we evoke here how other cerebral circuits might participate to this higher level cognitive architecture.

For each of these sensorimotor associations, there is not a unique relation between one stimulus and one action answering the corresponding question and the association can sometimes depend on a context, whatever its spatial or temporal form. This is the role of reinforcement learning, as compared to a simple hebbian associative rule : it can learn the best action to trigger to optimize reinforcement, depending on a state not only corresponding to a stimulus but to the aggregation of more information and also in the past and the future (Niv, 2009). The basal ganglia has often been described as a structure where a certain kind of reinforcement learning can take place (Joel, Niv, \& Ruppin, 2002), with its unique combination of afferences from cortical and extracortical structure and dopaminergic projections often reported to carry reward prediction errors, central in such algorithms. It is consequently noticeable that these circuits have been broken down in five parallel loops (Alexander, DeLong, \& Strick, 1986), with four loops directly addressing the four questions mentioned above (and receiving projections from the corresponding regions) and the last one for associative integration (cf figure 2 for more details).

More precisely, in the motor loop (how?), the dorsolateral striatum integrates information from the motor cortex and proprioceptive information from the sensory cortex. The oculomotor loop (Hikosaka, Nakamura, \& Nakahara, 2006) is related to the frontal eye field area for selection of gaze orientation (where?). The anterior cingulate loop includes the ventral striatum (mainly the core part of the nucleus accumbens) and is reported to energize the action, depending on the level of motivation and the cost of the action (Niv, 2007) (why?). The orbitofrontal loop includes the shell of the nucleus accumbens and is concerned with the hedonic value of objects (Kringelbach, 2005) (what?). For each of these loops, the role of the basal ganglia is to learn to trigger, depending on the context, different kinds of actions represented in the related region of the frontal cortex, as it is sketched in figure 2

A frequent situation occuring in realistic cases is that several objects in the environment can elicit several actions in the same or in different loops and that a global analysis has to be made to select the most advantageous action. The architecture of the basal ganglia has also been described as particularly adapted to this problem (Redgrave, Prescott, \& Gurney, 1999). In contrast to other structures like the cortex with distributed and sparse connectivity, the process of action selection in the basal ganglia is centralized and it can address conflicts between different loops. This is due to its funneling architecture providing a convergence of information (Parent \& Hazrati, 1995) and to other characteristics like a spiraling effect providing communications between the loops (Haber, Fudge, \& McFarland, 2000).

Among the loops, those answering to the questions What and Why have a specific role, because they address directly the problem of goal selection from internal needs and external opportunities, whereas questions how and where are more related to spatial contingencies (cf figure 3 for a more behavioral interpreta- 
tion). These limbic loops are particularly the place for articulation between pavlovian and operant conditioning, giving a special role to the shell and the core of the nucleus accumbens, the two input regions of the basal ganglia for these loops (Mannella, Gurney, \& Baldassarre, 2013). It is consequently important to notice that the hippocampus is mainly associated to these two loops (Voorn, Vanderschuren, Groenewegen, Robbins, \& Pennartz, 2004) (and not the others), providing episodic memories to the process of action selection of the basal ganglia. This has been analyzed as a key component of goal-directed behavior related to the evocation of specific cases, as compared to more habitual behavior rather observed in the other loops (Packard \& Knowlton, 2002).

The development of the prefrontal cortex has provided a more complex processing of time, with the development of working memory processes (O'Reilly, 2010), particularly useful in the limbic loops (what and why) to estimate histories of past experiences and contribute to a more accurate evaluation of uncertainty (McClure, Gilzenrat, \& Cohen, 2006). Considering more recent steps in the evolution process, the development of the prefrontal cortex is a major event and provides cognitive control to the intellectual abilities (Badre, 2008). This is another way to associate the different loops, by elaborating more complex rules to trigger actions. Even if this is described in terms of planning and reasoning, it remains anchored in the same scheme of loops between the basal ganglia and the prefrontal cortex.

\section{Discussion}

In this paper, we have described brain structures and functions within a global framework of information representation. Starting from a simple agent learning to satisfy some needs in an unknown environment, we have specified some constraints concerning information flows and learning principles and have associated these constraints to a general picture of the brain describing four main loops answering four questions important for survival. Then, we have begun to explain that some important cognitive capabilities extending beyond survival considerations are tightly linked to these principles because they have been elaborated and developed on this primitive substratum. The limited size of the paper prevented from going in more details on these cognitive functions and neither to extend to other functions related to the language for example but this should be done in future works. For the moment, the main behavioral interpretations of the framework elaborated here are summarized in figure 3 .

Several authors have already described the brain as answering such important questions, including (Ungerleider \& Mishkin, 1982) for the what/where opposition, (Milner \& Goodale, 1995) for the how and (Verschure, Pennartz, \& Pezzulo, 2014) for adding the why but these works were less accurate concerning the reciprocal mapping of brain structures and functions and the corresponding refinement along evolution. This idea is reminiscent of the principle of imbricated sensorimotor loops proposed in (Guillery, 2005), convenient both for incremental learning and for responding at anytime.

This work can be useful in computational neuroscience because it proposes a global framework where to include some specific model and can prevent from building models in isolation with no references to other structures, functions and related information flows and learning principles. It can also be useful in machine learning because it can be a source of inspiration for bio-inspired learning algorithms, particularly considering the problem of learning in autonomy. It can be consequently interesting in the domain of autonomous robotics, also because it emphasizes the important role of the body and the environment in the development of cognitive functions in the brain.

\section{Références}

Alexander, G., DeLong, M., \& Strick, P. (1986). Parallel organization of functionally segregated circuits linking basal ganglia and cortex. Ann. Rev. Neurosci., 9, 357-381.

Badre, D. (2008). Cognitive control, hierarchy, and the rostro-caudal organization of the frontal lobes. Trends in Cognitive Sciences, 12(5), 193-200.

Balleine, B. W., \& Killcross, S. (2006). Parallel incentive processing : an integrated view of amygdala function. Trends Neurosci, 29(5), 272-279. doi: 10.1016/j.tins.2006.03.002

Bandler, R., \& Shipley, M. T. (1994). Columnar organization in the midbrain periaqueductal gray : modules for emotional expression? Trends in Neurosciences, $17(9)$, 379-389.

Belova, M. A., Paton, J. J., Morrison, S. E., \& Salzman, C. D. (2007). Expectation modulates neural responses to pleasant and aversive stimuli in primate amygdala. Neuron, 55(6), 970-984.

Cardinal, R. N., Parkinson, J. A., Hall, J., \& Everitt, B. J. (2002). Emotion and motivation : the role of the amygdala, ventral striatum, and 


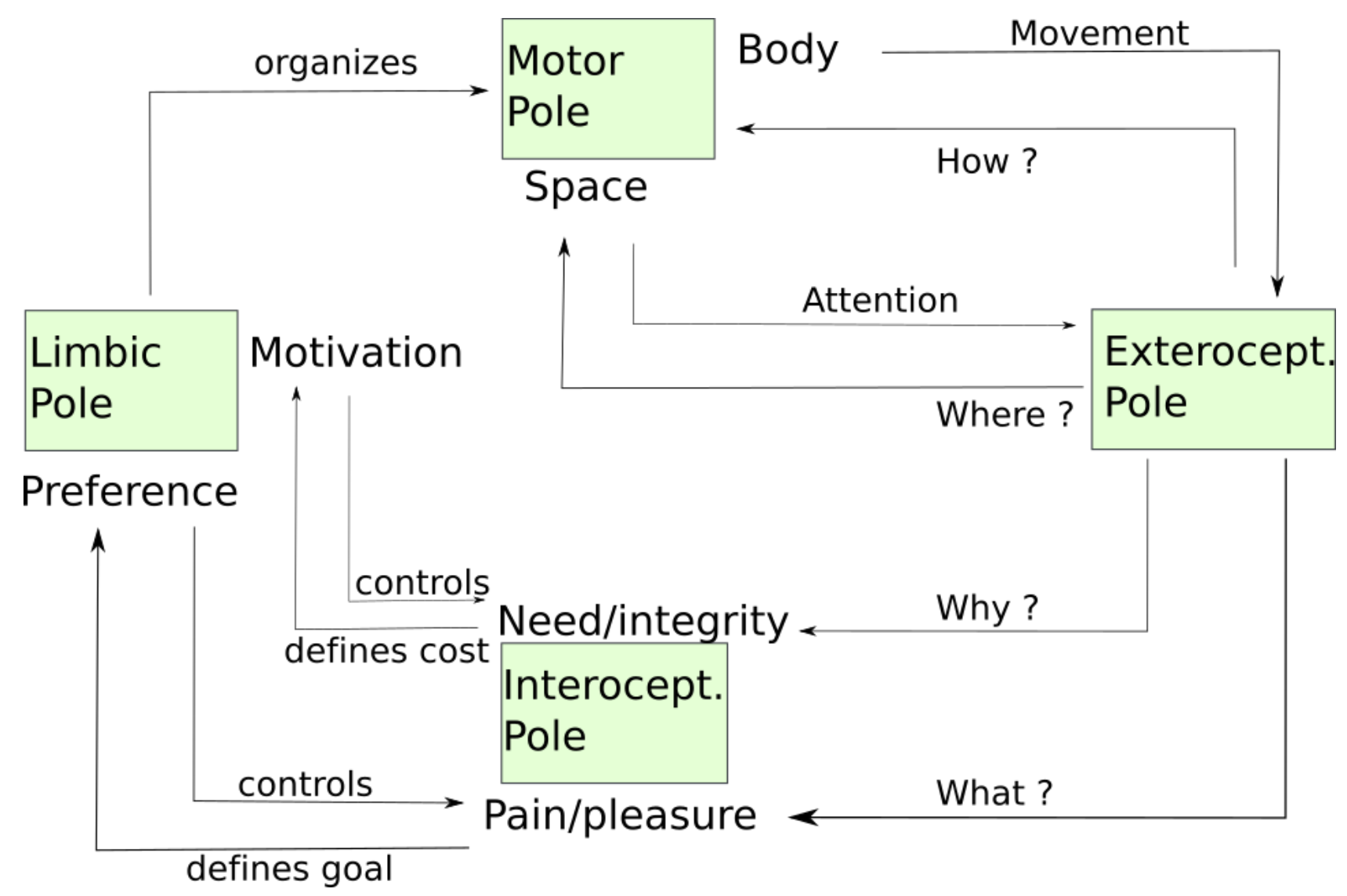

FIGURE 3 - This figure intends to integrate several lines of discussion in this paper, including the brain-bodyenvironment system and the framework of brain organization related to the four fundamental questions. We show in this figure that sensory interoceptive and exteroceptive poles interact with the limbic and motor poles to decide for the main characteristics of the behavior. Answers to the fundamental questions specify sensory constraints in the motor pole related to the position in space and to the body, and in the interoceptive pole related to pain and pleasure and to fundamental needs. Preferences and motivations in the limbic pole generate a consummatory behavior or organize a preparatory behavior with the motor pole that can trigger movements and evoke selective attention to obtain desired changes in the internal and external world and, accordingly, in the sensory perceptions. 
prefrontal cortex. Neuroscience \& Biobehavioral Reviews, 26(3), 321-352. doi: 10.1016/s0149 $-7634(02) 00007-6$

Carrere, M., \& Alexandre, F. (2015). A pavlovian model of the amygdala and its influence within the medial temporal lobe. Frontiers in Systems Neuroscience, 9(41).

Craig, A. (2003). Interoception : the sense of the physiological condition of the body. Current Opinion in Neurobiology, 13(4), 500-505.

Craig, A. (2009). How do you feel - now? the anterior insula and human awareness. Nat. Rev. Neurosci., 10, 59-70.

Damasio, A. R., \& Carvalho, G. B. (2013). The nature of feelings : Evolutionary and neurobiological origins. Nature Reviews Neuroscience, 14(2), 143-152.

Dean, P., Redgrave, P., \& Westby, G. W. (1989). Event or emergency? Two response systems in the mammalian superior colliculus. Trends in neurosciences, 12(4), 137-147.

Gros, C. (2010). Cognition and Emotion : Perspectives of a Closing Gap. Cognitive Computation, 2(2), 78-85.

Gruber, A. J., \& McDonald, R. J. (2012). Context, emotion, and the strategic pursuit of goals : Interactions among multiple brain systems controlling motivated behaviour. Frontiers in Behavioral Neuroscience, 6(50).

Guillery, R. W. (2005). Anatomical pathways that link perception and action. In Progress in brain research (Vol. 149, pp. 235-256). Elsevier.

Haber, S., Fudge, J., \& McFarland, N. (2000). Striatonigrostriatal pathways in primates form an ascending spiral from the shell to the dorsolateral striatum. The Journal of Neuroscience, 20(6), 2369-2382.

Hikosaka, O., Nakamura, K., \& Nakahara, H. (2006). Basal Ganglia orient eyes to reward. J. Neurophysiol, 95(2), 567-584.

Joel, D., Niv, Y., \& Ruppin, E. (2002). Actor-critic models of the basal ganglia : new anatomical and computational perspectives. Neural Networks, 15(4-6), 535-547.

Kringelbach, M. L. (2005). The human orbitofrontal cortex : linking reward to hedonic experience. Nat Rev Neurosci, 6(9), 691-702.

Laberge, F., Muhlenbrock-Lenter, S., Grunwald, W., \& Roth, G. (2006). Evolution of the Amygdala : New Insights from Studies in Amphibians. Brain Behav Evol, 67(4), 177-187. Consulté sur http://dx.doi.org/10.1159/000091119
Laborit, H. (1976). Eloge de la fuite. Folio Essais.

LeDoux, J. (2007). The amygdala. Current Biology, 17(20), R868-R874.

Lee, C., Rohrer, W. H., \& Sparks, D. L. (1988). Population coding of saccadic eye movements by neurons in the superior colliculus. Nature, 332(6162), 357-360. doi: 10.1038/332357a0

Mannella, F., Gurney, K., \& Baldassarre, G. (2013). The nucleus accumbens as a nexus between values and goals in goal-directed behavior : a review and a new hypothesis. Frontiers in behavioral neuroscience, 7 .

Manto, M., Bower, J. M., Conforto, A. B. B., DelgadoGarcía, J. M., da Guarda, S. N. F. N., Gerwig, M., ... Timmann, D. (2012). Consensus paper : roles of the cerebellum in motor control-the diversity of ideas on cerebellar involvement in movement. Cerebellum (London, England), 11(2), 457-487.

McClure, S., Gilzenrat, M., \& Cohen, J. (2006). An exploration-exploitation model based on norepinepherine and dopamine activity. In Y. Weiss, B. Schölkopf, \& J. Platt (Eds.), Advances in neural information processing systems 18 (pp. 867-874). MIT Press. Consulté sur http://www.csbmb.princeton.edu/ $\sim\{\}$ smcclure/pdf/MGC_NIPS.pdf

Middleton, F. A., \& Strick, P. L. (2000). Basal ganglia and cerebellar loops : motor and cognitive circuits. Brain Research Reviews, 31 (2), 236-250.

Milner, A., \& Goodale, M. (1995). The visual brain in action. Oxford University Press, USA.

Niv, Y. (2007). Cost, Benefit, Tonic, Phasic : What Do Response Rates Tell Us about Dopamine and Motivation. Annals of the New York Academy of Sciences, 1104 (1), 357-376.

Niv, Y. (2009, juin). Reinforcement learning in the brain. Journal of Mathematical Psychology, 53(3), 139-154. Consulté sur http://linkinghub.elsevier.com/retrieve/ pii/S0022249608001181

O'Regan, J. K., \& Noë, A. (2001). A sensorimotor account of vision and visual consciousness. The Behavioral and brain sciences, 24(5).

O'Reilly, R. C. (2010). The What and How of prefrontal cortical organization. Trends in Neurosciences, 33(8), 355-361.

Packard, M. G., \& Knowlton, B. J. (2002). Learning and memory functions of the basal ganglia. Annual review of neuroscience, 25 (1), 563-593.

Parent, A., \& Hazrati, L. N. (1995). Functional anatomy of the basal ganglia. I. The cortico-basal 
ganglia-thalamo-cortical loop. Brain Res Brain Res Rev, 20(1), 91-127.

Parvizi, J. (2009). Corticocentric myopia : old bias in new cognitive sciences. Trends in Cognitive Sciences, 13(8), 354-359.

Pessoa, L., \& Adolphs, R. (2010). Emotion processing and the amygdala : from a 'low road' to 'many roads' of evaluating biological significance. $\mathrm{Na}$ ture reviews. Neuroscience, 11 (11), 773-783. doi: $10.1038 / \mathrm{nrn} 2920$

Pfeifer, R., Bongard, J., \& Grand, S. (2007). How the body shapes the way we think: a new view of intelligence. MIT Press.

Redgrave, P., Prescott, T. J., \& Gurney, K. (1999). The basal ganglia : A vertebrate solution to the selection problem? Neuroscience, 89(4), 10091023.

Rousselet, G., Thorpe, S., \& Fabre-Thorpe, M. (2004). How parallel is visual processing in the ventral path? Trends in Cognitive Sciences, 8(8), 363370.

Sommer, M., \& Wurtz, R. (2004). What the brain stem tells the frontal cortex. I. Oculomotor signals sent from superior colliculus to frontal eye field via mediodorsal thalamus. Journal of neurophysiology, 91 (3), 1381-1402.

Swanson, L. W., \& Petrovich, G. D. (1998). What is the amygdala? Trends in Neurosciences, 21(8), 323-331.

Taouali, W., Goffart, L., Alexandre, F., \& Rougier, N. P. (2015). A parsimonious computational model of visual target position encoding in the superior colliculus. Biological Cybernetics, 109(4)(8), 549-559.

Ungerleider, L., \& Mishkin, M. (1982). Two cortical visual systems. In D. Ingle, M. Goodale, \& R. Mansfield (Eds.), Analysis of visual behavior (pp. 549-586). MIT Press.

Varela, F., Thompson, E., \& Rosch, E. (1991). The embodied mind : Cognitive science and human experience. Cambridge, MA : MIT Press.

Verschure, P. F. M. J., Pennartz, C. M., \& Pezzulo, G. (2014). The why, what, where, when and how of goal-directed choice : neuronal and computational principles. Philosophical Transactions of the Royal Society B : Biological Sciences, 369(1655), $20130483+$.

Voorn, P., Vanderschuren, L. J., Groenewegen, H. J., Robbins, T. W., \& Pennartz, C. M. (2004). Putting a spin on the dorsal-ventral divide of the striatum. Trends Neurosci, 27(8), 468-474. 